\title{
Quantitative Expression Analysis of Circulating miRNAs Reveals Significant Association with Cardiovascular Pathogenesis in Women
}

\author{
Chandrakala Lakkireddy ${ }^{1}$ Siddharth Rout ${ }^{2} \quad$ Shaik lqbal Ahmed ${ }^{1} \quad$ Nooruddin Owaisi ${ }^{1}$ \\ Avinash Bardia ${ }^{1}$ Nagarapu Raju' $\quad$ Sandeep Kumar Vishwakarma ${ }^{1} \quad$ Aleem Ahmed Khan ${ }^{1}$ \\ ${ }^{1}$ Central Laboratory for Stem Cell Research and Translational \\ Medicine, Centre for Liver Research and Diagnostics, Deccan \\ College of Medical Sciences, Hyderabad, Telangana, India \\ ${ }^{2}$ Department of Cardiology, Owaisi Hospital and Research Centre, \\ Deccan College of Medical Sciences, Hyderabad, Telangana, India

\begin{abstract}
Address for correspondence Aleem Ahmed Khan, PhD, Central Laboratory for Stem Cell Research and Translational Medicine, Centre for Liver Research and Diagnostics, Deccan College of
\end{abstract} \\ Medical Sciences, Kanchanbagh, Hyderabad 500058, Telangana, \\ India (e-mail: aleem_a_khan@rediffmail.com).
}

Ind J Car Dis Wom 2020;5:8-14

\begin{abstract}
Keywords

- cardiovascular diseases

- circulating miRNAs

- troponin

- relative expression

Background Cardiovascular disease (CVD) represents a complex pathophysiological condition with continuous increasing mortality and poor treatment outcome. Troponin is the only standard biochemical indication of CVD, which fails to exactly determine the diagnostic and prognostic indications of CVD. Circulating microribonucleic acids (miRNAs) represent crucial molecular targets to determine the pathophysiology of CVD. Hence, in the present study quantitative estimation of three miRNAs (miRNA-133, miRNA-155, and miRNA-222) targeting different pathways of CVD pathogenesis has been performed to find their role.

Methods and Materials A total of 50 female subjects including 25 CVD patients and 25 controls with age range of 18 to 65 years were included in this study. The extraction of circulating miRNAs was done in all the serum samples separated through centrifugation. Relative expression of all three miRNAs was performed using SYBR green-based real-time polymerase chain reaction (RT-PCR) in comparison to internal control.

Results Significantly upregulated expression levels were observed for miRNA-133 and miRNA-222 in CVD women patients as compared with control subjects. Age-wise analysis revealed highly upregulated expression of miRNA-133 in patients having age group $<45$ years. While comparing with troponin levels, miRNA-222 was found significantly upregulated in patients having troponin levels $<250 \mathrm{ng} / \mathrm{L}$ whereas miRNA-133 was found significantly higher in $>250 \mathrm{ng} / \mathrm{L}$ troponin group.

Conclusion Circulating miRNAs specifically miRNA-133 and miRNA-222 have essential role in pathogenesis of CVD in women patients.
\end{abstract}

\section{Introduction}

Cardiovascular diseases (CVD) affect normal functions of blood vessels and heart which is limited to epicardial coronary arteries. Every year $>8$ million women die worldwide due to CVD, leading to one-third of deaths in females. ${ }^{1,2}$ Women with CVD have constant suboptimal treatment pattern with higher rate of mortality and poor outcome as compared with men. ${ }^{3,4}$ Mechanisms responsible for complex pathophysiology involved in development of CVDs are still unclear. Hence, a hypothetic alter ahead of anatomic portrayal of coronary artery disease is required to interpret CVD risk identification and for designing effective treatment strategies for women. Moreover, there is a need to identify new diagnostic biomarkers and prognostic factors to detect, prevent, and for proper treatment of women with CVD.

Currently, the most commonly used biomarker for CVD is cardiac troponins due to its higher levels of sensitivity and 
specificity in cardiac necrosis. ${ }^{5}$ However, recent medical advances have showed essential role of epigenetic regulatory element-like circulatory microribonucleic acids (miRNAs) as potent biomarker to define the disease pathogenesis at molecular levels. These miRNAs are the most widely studied noncoding ribonucleic acid (RNA) sequences of $\sim 21$ to 25 nucleotides in size. It has crucial role in transcriptional regulation of gene expression of cellular physiological mechanisms such as proliferation, differentiation, and immunological responses, and so forth, directly or indirectly. ${ }^{6}$ miRNAs are highly stable in circulation; hence, it has been proposed for investigating their potential as noninvasive genetic markers of high degree of sensitivity and specificity with rapid and accurate detection of disease pathogenesis..$^{7-9}$ Thus, evaluation of functional miRNAs profile in CVD may explore their useful potential as better molecular diagnosis criteria.

In this study, we have quantified the levels of three circulating miRNAs (miRNA-133, miRNA-155, miRNA-222) involved in different pathways in women with CVD. Among different regulatory elements, miRNA-133 is one of the most abundant miRNAs in cardiac tissues that inhibits proliferation of cardiomyocytes. ${ }^{10-12}$ It is also a critical regulator of cardiac and skeletal muscles development and has crucial role during pathological alterations and vascular smooth muscle cell biology. ${ }^{12}$ The second miRNA, miRNA-155, has been reported as one of the most potent prognostic biomarker for myocardial infarction patients with cardiac death. ${ }^{13}$ It is also a crucial pleiotropic regulator of cellular homeostasis, inflammatory mechanisms, viral infections, hematopoietic cell lineage differentiation, and cardiac malfunction. ${ }^{14,15}$ While, miRNA-222 play protective role against pathogenetic cardiac proliferation, migration, remodeling, and apoptosis of smooth muscle cells., ${ }^{9,16,17}$ It also controls physiological functions of cardiac cells and its abnormal expression has been attributed with several types of CVDs. Therefore, quantitative evaluation of these three circulating miRNAs may provide crucial information of genetic basis of CVD pathogenesis in Indian women for better diagnosis, prognosis, and identifying suitable molecular targets for designing effective treatment strategies.

\section{Materials and Methods}

This study was performed after getting institutional review board (IRB) approval from of Deccan college of Medical Sciences, Hyderabad, Telangana, India. Signed informed consent forms (CRF) were collected from all the subjects included in the study who were attending Department of Cardiology, Owaisi Hospitals and Research Centre, Hyderabad, Telangana, India.

\section{Study Population}

Totally 50 subjects ( 25 female CVD patients and 25 female controls) were included in this study. Blood samples were collected from all the female subjects within the age group of 18 to 65 years. Demographical description of every subject was recorded in sample collection proforma.

\section{Sample Collection and Processing}

Peripheral blood sample of $3 \mathrm{~mL}$ was withdrawn from each subject and transferred in serum collection tubes. Following to sample collection, all serum tubes were incubated for 15 minutes at room temperature for proper coagulation. Further serum separation was done by centrifugation at $3,000 \mathrm{rpm}$ for 15 minute at room temperature. Further miRNA extraction was performed immediately from the separated serum to avoid degradation of miRNAs.

\section{Extraction of Serum miRNAs}

Serum miRNAs extraction was performed within 4 hours of serum separation using miRNeasy Serum/Plasma Kit (Qiagen, Cat No./ID: 217184). In brief, $100 \mu \mathrm{L}$ of serum sample from each patient was lysed and loaded into a column containing specific nitrocellulose membrane filters for entrapment of miRNAs. Wash buffers were used to wash off all impurities and finally pure membrane bound miRNAs were eluted using highly concentrated elution buffer supplied with the kit.

\section{Preparation of Complementary DNA}

All the extracted miRNAs were used for complementary DNA (cDNA) conversion into a thermocycler. Universal stem loop primer (USLP) and reverse transcription (RT) primers were used for cDNA preparation as described earlier. ${ }^{18}$ Briefly, reaction mixture of extracted miRNAs with USLP was initially incubated at $65^{\circ} \mathrm{C}$ for 10 minutes followed with 2 minutes of snap cooling in ice. Furthermore, reaction components such as deoxyribonucleotide triphosphates (dNTPs), reverse transcription-polymerase chain reaction (RT-PCR) buffer, and reverse transcriptase (RTase) enzyme were mixed along with diethyl pyrocarbonate (DEPC)-treated millique water to complete the reaction volume and incubated at $42^{\circ} \mathrm{C}$ for one hour followed by $72^{\circ} \mathrm{C}$ for 15 minutes of incubation. Finally, prepared cDNA was stored at $-20^{\circ} \mathrm{C}$ and further selected miRNAs quantification was performed by using sequence specific primers.

\section{Quantification of Selected miRNAs Levels using Quantitative Real-time Polymerase Chain Reaction}

Quantification was performed by SYBR-Green assay using quantitative real-time polymerase chain reaction (qRT-PCR) (ABI 7500, Applied Biosystems) for selected miRNAs [(miR-133 (FP-TTTGGTCCCCTTCAACCAGCTG; RP-GCGAGCACAGAATTAATACGAC), miR-155 (FP-CTAGCCTGCAGGTATTCAAATATTTCCACAGA; RP-ATCCGGCCGGCCTGAAGATGGTTATGAACATA), miR-222 (FP-GCTGCTGGAAGGTGTAGGTA; RP- GATGCCATCAGAGACCCAGT) U6 SnRNA FP-CGCTTCGGCAGCAGCACATATACTA; RP- CGCTTCACGAATTTGCGTGTCA)]. To evaluate expression levels in each sample, quantification was done by using specific set of human miRNA forward and reverse primers. For qRT-PCR, initial denaturation was done for 2 minutes at $95^{\circ} \mathrm{C}$ which was followed by 40 cycles of denaturation step for 30 second at $94^{\circ} \mathrm{C}$, followed by annealing for 30 seconds at $58^{\circ} \mathrm{C}$ and extension for 30 seconds at $72^{\circ} \mathrm{C}$. At the end of PCR cycles 10 minute of melting curve analysis was set to differentiate between primer-dimer and amplicons. All the reactions were performed in triplicates for each set of miRNA and mean 
values were used for further calculations. Relative fold change was used to quantify the expression levels of each miRNA in control as well as CVD female subjects with respect to the quantity of U6 SnRNA present in the same sample.

\section{Statistical Analysis}

Relative quantification method $\left(R Q=2^{-\Delta \Delta C T}\right)$ as described by Livak $^{19}$ was used to calculate the changes in expression levels of each miRNA. Column analysis was performed using $t$-test to determine the significant difference between the control subjects and CVD patients. Furthermore, one way ANOVA was done to identify significant difference among multiple groups. All the analysis was performed using Graph Pad Prism software (Version V) and statistical significance was set at $p$ value $\leq 0.05$.

\section{Results}

Expression Levels of Circulating miRNA-133, miRNA-155, and miRNA-222 in CVD Female Patients

Quantitative expression analysis of miRNAs in serum samples of CVD patient's in comparison to control female subjects demonstrated significant upregulation of miRNA-133 (confidence interval $[\mathrm{CI}]:-0.6817$ to $-0.1484, p=0.005$ ) (-Fig. 1a) and miRNA-222 (CI: -5.918 to $-1.199, p=0.007$ ) (-Fig. 1c). Another circulatory miR-155 which was found to have a significant role in inflammatory signal transduction couldn't show significant change in CVD female patients as compared with female control subjects (CI: -0.7419 to $0.09780, p>0.05$ ) (-Fig. 1b). Furthermore, correlation of all three miRNAs revealed significant upregulation of miRNA-133 as compared with miRNA-155 (CI: 2.303-66.83, $p<0.05$ ) and miRNA-222 (CI: 0.7400-67.39, $p<0.05$ ) (-Fig. 1d).

\section{Age-wise Changes in Expression Levels of miRNAs in CVD Female Patients}

For analyzing age-wise changes in levels of expression of non-coding RNAs, all CVD female patients were categorized into two groups: $<45$ years and $>45$ years. The relative quantification data revealed that miRNA-133 has significantly higher expression levels than miRNA-155 (CI: 42.42-117.7, $p=0.0001)$ and miRNA-222 (CI: 40.40-110.1, $p=0.0001$ ) in CVD patients with $<45$ years of age ( - Fig. 2a). Similarly, miRNA-133 expression levels were also increased significantly than miRNA-155 (CI: 5.250-20.37, $p=0.001$ ) and miRNA-222 (CI: 1.856-18.60, $p=0.01$ ) in CVD female patients a
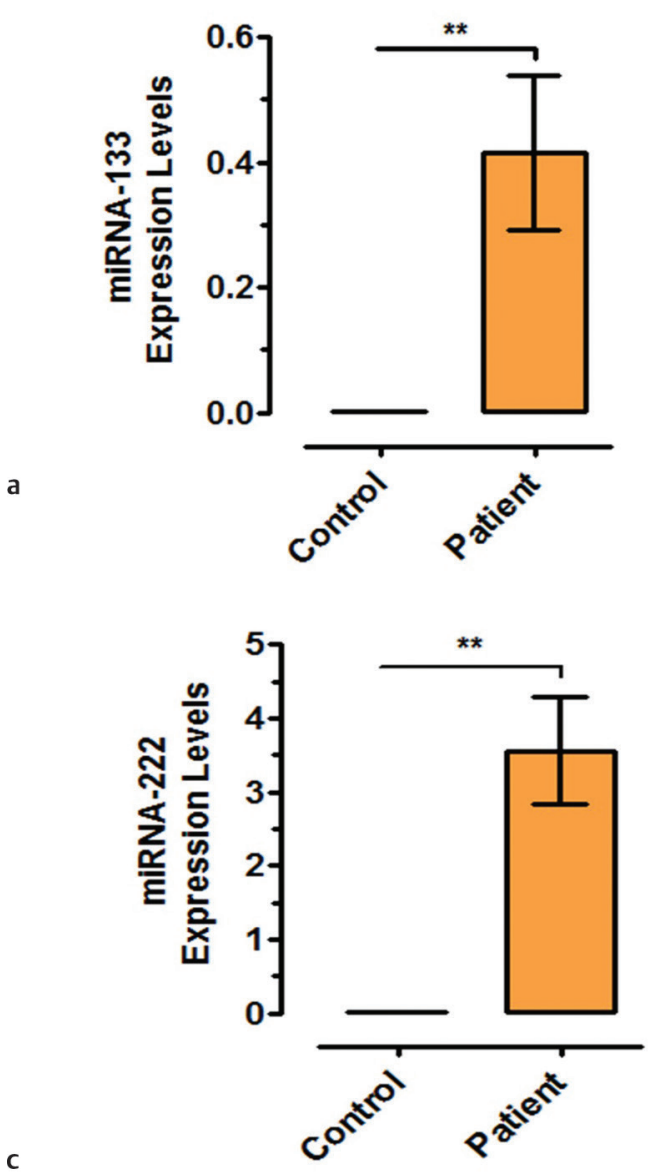
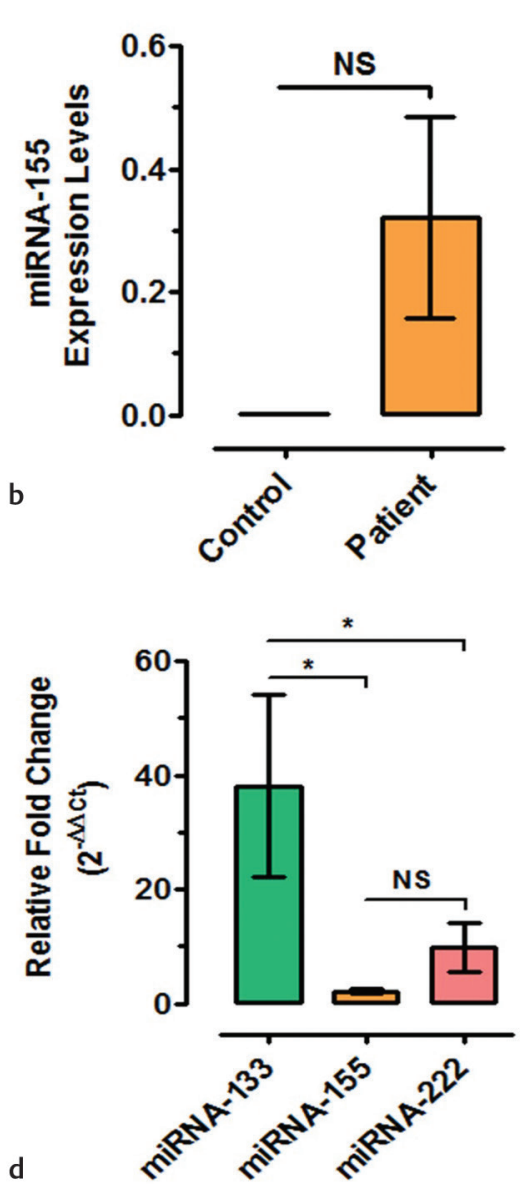

Fig. 1 Changes in expression levels of different microribonucleic acids (miRNAs) in cardiovascular disease (CVD) patients as compared with control subjects $\left({ }^{*} p=0.01,{ }^{* *} p=0.001\right.$, NS $=$ not significant $)$. 

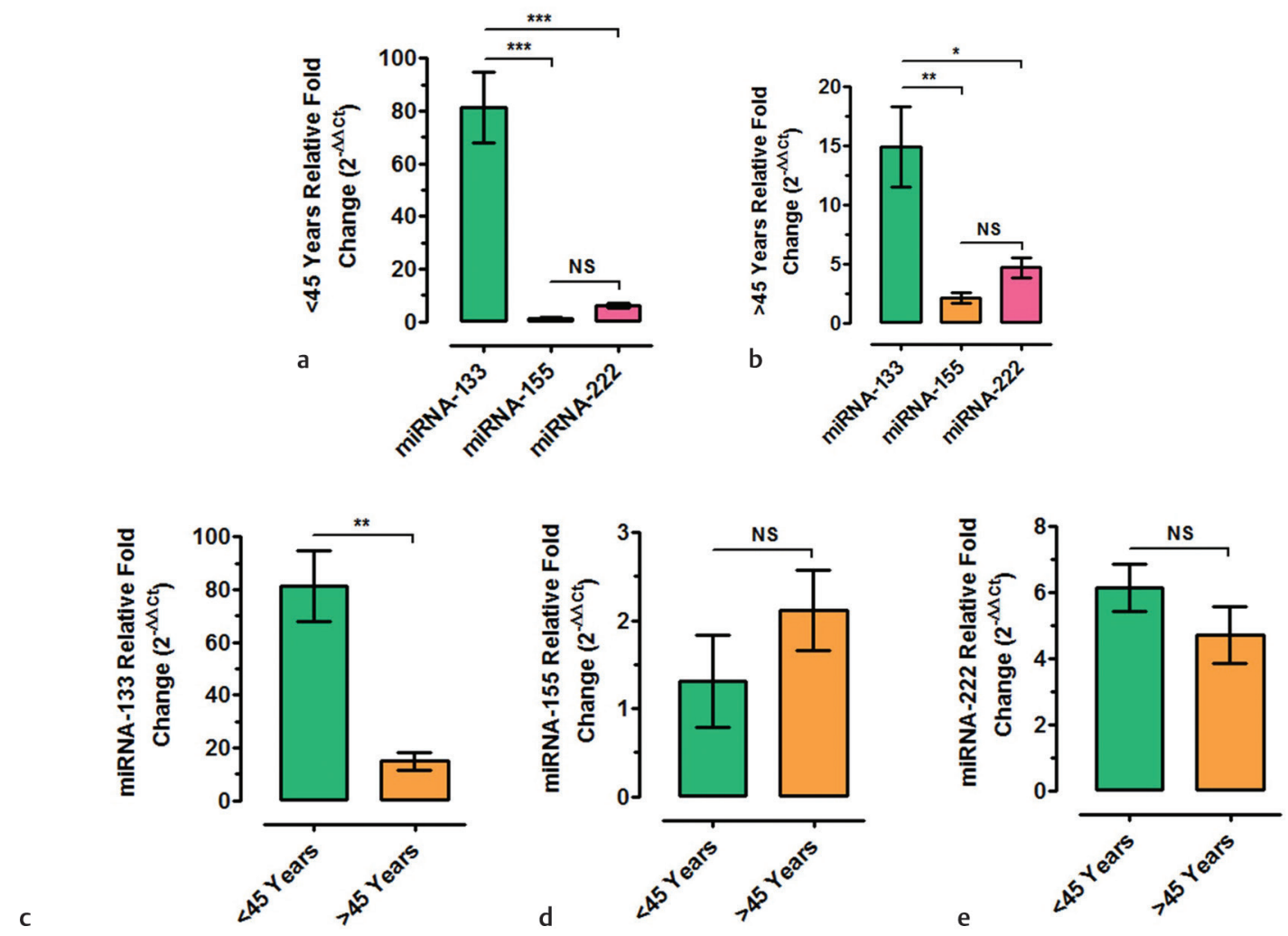

Fig. 2 Age-wise changes in relative expression levels of different microribonucleic acids (miRNAs) in cardiovascular disease (CVD) female patients $\left({ }^{*} p=0.01,{ }^{*} p=0.001,{ }^{* * *} p=0.0001, \mathrm{NS}=\right.$ not significant $)$.

of $>45$-year age group ( - Fig. $2 \mathbf{b}$ ). However, no significant difference was observed for miRNA-155 as compared with miRNA-222 $(p>0.05)$ in both the age group of patients.

Furthermore, comparative analysis of individual miRNA in both the age groups revealed that miRNA-133 has significantly higher level of expression in CVD patients with $<45$ years as compared with $>45$-year-old patients (CI: 37.35-95.55, $p=0.001$, - Fig. 2c), while no significant difference was observed for miRNA-155 and miRNA-222 during age-wise comparison ( $p>0.05,-$ Fig. 2 d,e).

\section{Correlative Expression Levels of miRNA-133,} miRNA-155, and miRNA-222 in CVD Female Patients Based on Troponin Levels

Relative quantitative expression analysis of three chosen miRNAs was analyzed based on the troponin levels of CVD female patients into two categories $<250 \mathrm{ng} / \mathrm{L}$ of troponin and $>250 \mathrm{ng} / \mathrm{L}$ troponin ( - Fig. 3). The expression analysis revealed that miRNA-222 had significantly higher expression levels in patients having troponin levels $<250 \mathrm{ng} / \mathrm{L}$ as compared with miRNA-133 (CI: -3.854 to $-1.249, p=0.0001$ ) and miRNA-155 (CI: -4.427 to $-1.313, p=0.0001$ ), whereas no significant difference was observed among the expression levels of miRNA-133 and miRNA-155 ( $p>0.05)$ in CVD female patients with $<250 \mathrm{ng} / \mathrm{L}$ of troponin levels ( - Fig. 3a). In contrast to these results, CVD female patients having troponin levels $>250 \mathrm{ng} / \mathrm{L}$ showed significantly upregulated expression of miRNA-133 as compared with miRNA-155
(CI: 8.752-96.85, $p=0.01$ ) and miRNA-222 (CI: 14.38-93.91, $p=0.001$ ), respectively, while no significant difference in relative expression levels was observed between miRNA-155 and miRNA-222 $(p>0.05)$ ( - Fig. $3 \mathbf{b})$. These findings are well reflected in correlation coefficient plots of miRNAs with serum troponin levels ( - Fig. $\mathbf{3 c}, \mathbf{d}$ ).

\section{Discussion}

Continuous increasing mortality rate and poor treatment outcome of CVD women patients as compared with men has posed a crucial need to identify suitable diagnosis and prognostic information or biomarker. The identification of circulating regulatory molecules in peripheral blood has led to a new meadow of investigation which is involved in complex pathophysiological mechanisms for the development of several types of cardiovascular diseases. ${ }^{20-23}$

Among different circulating miRNAs identified till date, miRNA-133 has shown a crucial role in CAD patients. ${ }^{24-26}$ Studies have demonstrated that overexpression of miRNA-133 leads to preserved cardiac functions and inhibition of miRNA-133 expression results in increased hypertrophy. ${ }^{27}$ It is highly abundant in cardiac cells wherein its manipulation affects cardiac action potential. ${ }^{28}$ In our study, we observed significantly upregulated expression levels of circulating miRNA-133 levels in CVD women patients as compared with control subjects (-Fig. 1a). Furthermore, as compared with other miRNAs tested in this study, 

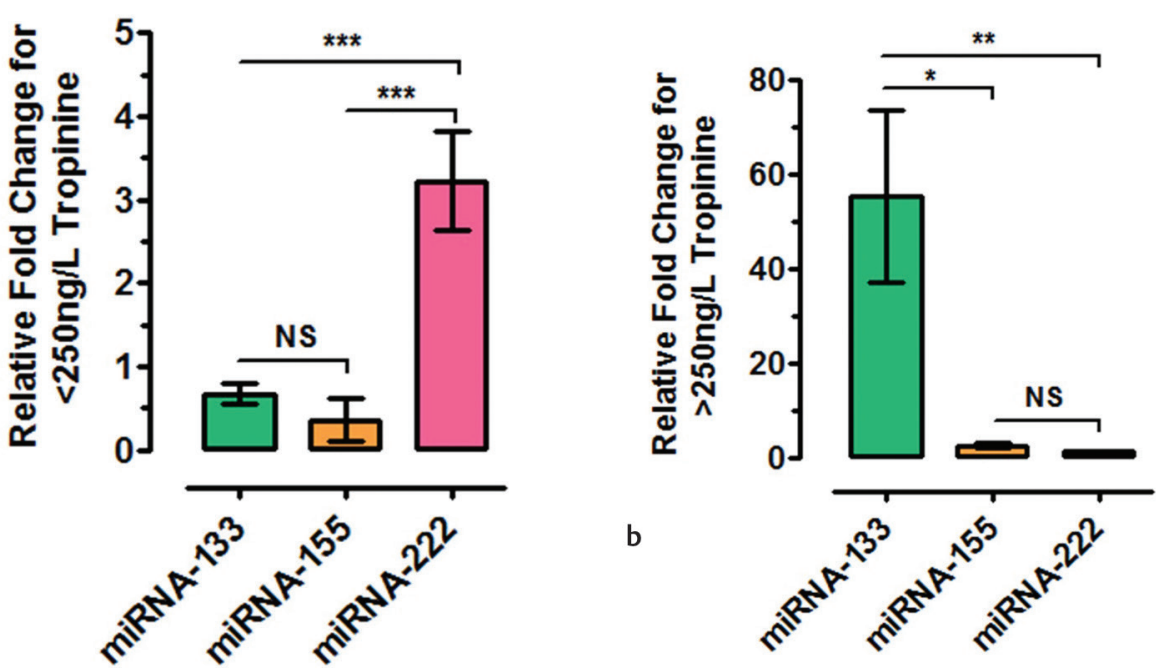

a

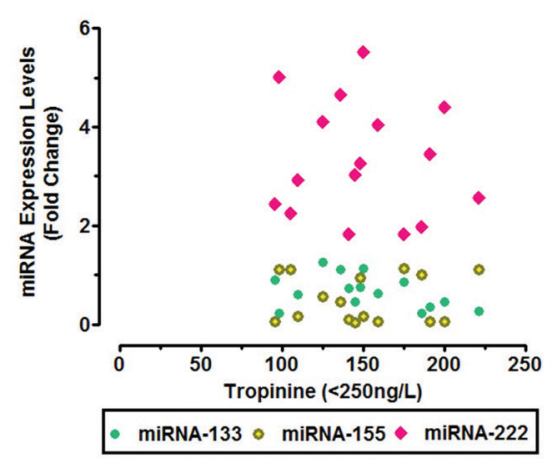

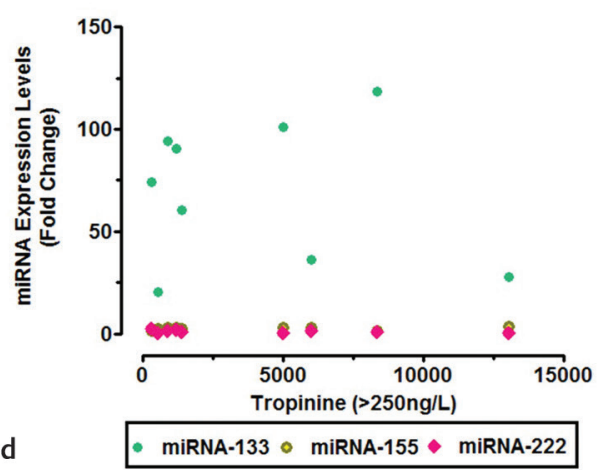

Fig. 3 Correlative expression levels of miRNA-133, miRNA-155 and miR-222 in cardiovascular disease (CVD) female patients based on two different categories of troponin levels $\left({ }^{*} p=0.01,{ }^{* *} p=0.001,{ }^{* * *} p=0.0001\right.$, NS $=$ not significant).

miRNA-133 level was significantly higher in CVD patients revealing that miRNA-133 play crucial role in pathophysiological mechanisms involved in CVD. In addition to the function of miRNA-133 in cardiac hypertrophy, it is also involved in antifibrotic mechanisms through connective tissue growth factors targeting which were considered as key regulators of fibrotic processes, the major constituents of cardiac extracellular matrix (ECM). ${ }^{29,30}$ Hence, we also investigated, the role of miRNA-133 based on age in CVD women patients which has important role in cardiac ECM remodeling. Age-wise data revealed that miRNA-133 is significantly higher in patients with < 45 years of age as compared with older patients (-Fig. 2c). This data signifies involvement of miRNA-133 in ECM remodeling of cardiac vessels and pathogenesis of CVD in women.

Another basic mechanism of CVD pathogenesis involved inflammatory regulators. In this regard miRNA-155 has been observed to be upregulated during cardiac pathogenesis. ${ }^{30-32}$ This particular miRNA play role as proarteriogenic and antiangiogenic factor in cardiac diseases. ${ }^{33}$ Hence, we studied expression levels of circulating miRNA-155 in CVD women patients. Results demonstrated that miRNA-155 does not have any significant effect of CVD pathogenesis or age factor or troponin levels. This could be explained as the patients enrolled in this study may have passed initial mechanism of inflammatory signals initiated during CVD pathogenesis and more toward the ECM and cellular modifications.

The third type of circulating miRNA (miRNA-222) is significantly involved in promoting the cardiac hypertrophy, expansion, and survival mechanisms. ${ }^{34}$ It also plays a protective role during cardiac dysfunction due to ischemic injury, and heart development. ${ }^{16,35}$ In our study, we have observed significantly upregulated expression levels of miRNA-222 in CVD women patients in comparison to control subjects (-Fig. 1c); however, upregulation of miRNA-222 was significantly at lower side as compared with miRNA-133 (-Fig. 1d). Although, age-wise comparison of miRNA-22 did not show significant change, it was significantly higher in patients with troponin levels $<250 \mathrm{ng} / \mathrm{L}$ (-Fig. 3a), which was further reduced as compared with miRNA-133 in CVD women patients with troponin levels $>250 \mathrm{ng} / \mathrm{L}$ ( $\mathbf{- F i g}$. $3 \mathbf{b}$ ). Thus, these data revealed that miRNA-222 has an essential role in CVD women patients having less troponin levels.

In summary, study has signified the crucial role of each miRNA; more specifically, miRNA-133 showed significant involvement in CVD severity in women as observed with the higher troponin levels. Serum troponin levels have been considered as one of the biochemical parameters to define the severity of CVD in patients. Increase in 
the serum troponin levels directly reflects the increase in cardiac muscles damage, thereby increased severity of CVD. In this study, we have categorized CVD patients into two groups $-(1)<250 \mathrm{ng} / \mathrm{L}$ troponin levels and (2) $>250 \mathrm{ng} / \mathrm{L}$ troponin levels-to identify significance of miRNA expression with serum troponin levels for indirect correlation with CVD severity. Although the findings of this study are interesting and of high value, sample size and correlation with more clinical parameters represent major limitations of the study. Hence, large cohort studies including more number of patients and clinical parameters along with serum miRNA profiling could provide better outcome.

\section{Conclusion}

Results from this study showed that miRNA-133 and miRNA-222 have an essential role in the pathogenesis of CVD in women. Furthermore, miRNA-133 showed higher significance in CVD women patients of different ages as compared with miRNA-155 and miRNA-222. miRNA-222 was found significantly associated with CVD pathogenesis in women patients having troponin levels $<250 \mathrm{ng} / \mathrm{L}$, whereas miRNA-133 showed significant association with troponin levels >250 ng/L in CVD women.

\section{Conflict of Interest}

The authors have declared no conflict of interest.

\section{Acknowledgment}

None.

\section{References}

1 Global Atlas on Cardiovascular Disease Prevention and Control. Geneva, Switzerland: World Health Organization (in collaboration with the World Heart Federation and World Stroke Organization); 2011

2 Pilote L, Dasgupta K, Guru V, et al. A comprehensive view of sex-specific issues related to cardiovascular disease. CMAJ 2007;176(6):S1-S44

3 Daly C, Clemens F, Lopez Sendon JL, et al; Euro Heart Survey Investigators. Gender differences in the management and clinical outcome of stable angina. Circulation 2006;113(4):490-498

4 Vaccarino V, Parsons L, Peterson ED, Rogers WJ, Kiefe CI, Canto J. Sex differences in mortality after acute myocardial infarction: changes from 1994 to 2006. Arch Intern Med 2009;169(19):1767-1774

5 Heil B, Tang WH. Biomarkers: their potential in the diagnosis and treatment of heart failure. Cleve Clin J Med 2015;82(12, Suppl 2):S28-S35

6 Wahid F, Shehzad A, Khan T, Kim YY. MicroRNAs: synthesis, mechanism, function, and recent clinical trials. Biochim Biophys Acta 2010;1803(11):1231-1243

7 Weber JA, Baxter DH, Zhang S, et al. The microRNA spectrum in 12 body fluids. Clin Chem 2010;56(11):1733-1741

8 Zampetaki A, Kiechl S, Drozdov I, et al. Plasma microRNA profiling reveals loss of endothelial miR-126 and other microRNAs in type 2 diabetes. Circ Res 2010;107(6):810-817

9 Liu R, Zhang C, Hu Z, et al. A five-microRNA signature identified from genome-wide serum microRNA expression profiling serves as a fingerprint for gastric cancer diagnosis. Eur J Cancer 2011;47(5):784-791

10 Hata A. Functions of microRNAs in cardiovascular biology and disease. Annu Rev Physiol 2013;75:69-93

11 Zhao Y, Samal E, Srivastava D. Serum response factor regulates a muscle-specific microRNA that targets Hand2 during cardiogenesis. Nature 2005;436(7048):214-220

12 Chen JF, Mandel EM, Thomson JM, et al. The role of microRNA-1 and microRNA-133 in skeletal muscle proliferation and differentiation. Nat Genet 2006;38(2):228-233

13 Matsumoto S, Sakata Y, Nakatani D, et al. A subset of circulating microRNAs are predictive for cardiac death after discharge for acute myocardial infarction. Biochem Biophys Res Commun 2012;427(2):280-284

14 Faraoni I, Antonetti FR, Cardone J, Bonmassar E. miR-155 gene: a typical multifunctional microRNA. Biochim Biophys Acta 2009;1792(6):497-505

15 Elton TS, Selemon H, Elton SM, Parinandi NL. Regulation of the MIR155 host gene in physiological and pathological processes. Gene 2013;532(1):1-12

16 Liu X, Xiao J, Zhu H, et al. miR-222 is necessary for exercise-induced cardiac growth and protects against pathological cardiac remodeling. Cell Metab 2015;21(4):584-595

17 Celic T, Metzinger-Le Meuth V, Six I, Massy ZA, Metzinger L. The mir-221/222 cluster is a key player in vascular biology via the fine-tuning of endothelial cell physiology. Curr Vasc Pharmacol 2017;15(1):40-46

18 Yang LH, Wang SL, Tang LL, et al. Universal stem-loop primer method for screening and quantification of microRNA. PLoS One 2014;9(12):e115293

19 Livak KJ, Schmittgen TD. Analysis of relative gene expression data using real-time quantitative PCR and the $2(-\Delta \Delta \mathrm{C}(\mathrm{T}))$ method. Methods 2001;25(4):402-408

20 Liu N, Olson EN. MicroRNA regulatory networks in cardiovascular development. Dev Cell 2010;18(4):510-525

21 Small EM, Olson EN. Pervasive roles of microRNAs in cardiovascular biology. Nature 2011;469(7330):336-342

22 Jamaluddin MS, Weakley SM, Zhang L, et al. miRNAs: roles and clinical applications in vascular disease. Expert Rev Mol Diagn 2011;11(1):79-89

23 Hsu A, Chen SJ, Chang YS, Chen HC, Chu PH. Systemic approach to identify serum microRNAs as potential biomarkers for acute myocardial infarction. BioMed Res Int 2014;2014:418628

24 Carè A, Catalucci D, Felicetti F, et al. MicroRNA-133 controls cardiac hypertrophy. Nat Med 2007;13(5):613-618

25 Fichtlscherer S, De Rosa S, Fox $\mathrm{H}$, et al. Circulating microRNAs in patients with coronary artery disease. Circ Res 2010;107(5):677-684

26 Trajkovski M, Ahmed K, Esau CC, Stoffel M. MyomiR-133 regulates brown fat differentiation through Prdm16. Nat Cell Biol 2012;14(12):1330-1335

27 Castaldi A, Zaglia T, Di Mauro V, et al. MicroRNA-133 modulates the $\beta 1$-adrenergic receptor transduction cascade. Circ Res 2014;115(2):273-283

28 Matkovich SJ, Wang W, Tu Y, et al. MicroRNA-133a protects against myocardial fibrosis and modulates electrical repolarization without affecting hypertrophy in pressure-overloaded adult hearts. Circ Res 2010;106(1):166-175

29 Duisters RF, Tijsen AJ, Schroen B, et al. miR-133 and miR-30 regulate connective tissue growth factor: implications for a role of microRNAs in myocardial matrix remodeling. Circ Res 2009;104(2):170-178

30 Castoldi G, Di Gioia CR, Bombardi C, et al. MiR-133a regulates collagen 1A1: potential role of miR-133a in myocardial fibrosis in angiotensin II-dependent hypertension. J Cell Physiol 2012;227(2):850-856 
31 Kin K, Miyagawa S, Fukushima S, et al. Tissue- and plasma-specific MicroRNA signatures for atherosclerotic abdominal aortic aneurysm. J Am Heart Assoc 2012;1(5):e000745

32 Faccini J, Ruidavets JB, Cordelier P, et al. Circulating miR-155, miR-145 and let-7c as diagnostic biomarkers of the coronary artery disease. Sci Rep 2017;7:42916

33 Pankratz F, Bemtgen X, Zeiser R, et al. MicroRNA-155 Exerts Cell-Specific Antiangiogenic but Proarteriogenic Effects During Adaptive Neovascularization. Circulation 2015;131(18):1575-1589
34 Wei Y, Zhu M, Corbalán-Campos J, Heyll K, Weber C, Schober A. Regulation of Csf1r and Bcl6 in macrophages mediates the stage-specific effects of microRNA-155 on atherosclerosis. Arterioscler Thromb Vasc Biol 2015;35(4):796-803

35 Li D, Ji L, Liu L, et al. Characterization of circulating microRNA expression in patients with a ventricular septal defect. PLoS One 2014;9(8):e106318 